\title{
An observational study on the relationship between plasma vitamin C, blood glucose, oxidative stress, endothelial dysfunction and outcome in patients with septic shock
}

\author{
K G H Katundu, ${ }^{1}$ MSc (Med) Nutrition, MBBS; L T Hill, ${ }^{2} \mathrm{PhD}$ (Physiology), RD (SA); L M Davids, ${ }^{3} \mathrm{PhD}$ (Med), MSc (Med); \\ I A Joubert, ${ }^{4} \mathrm{MB}$ BCh, DA (SA), FCA (SA); M G A Miller, ${ }^{4} \mathrm{MB}$ ChB, DA (SA), FCA, Cert Crit Care (SA); \\ J L Piercy, ${ }^{4}$ BSc (Hons), MBBS (Lond), FCA (SA), Cert Crit Care (SA); W L Michell, ${ }^{2}$ MB ChB, DA (SA), FFA (SA) \\ ${ }^{1}$ Division of Human Nutrition, Department of Human Biology, Faculty of Health Sciences, University of Cape Town, South Africa \\ ${ }^{2}$ Division of Critical Care, Department of Surgery, Faculty of Health Sciences, University of Cape Town, South Africa \\ ${ }^{3}$ Redox Laboratory, Department of Human Biology, Faculty of Health Sciences, University of Cape Town, South Africa \\ ${ }^{4}$ Division of Critical Care, Department of Anaesthesia, Faculty of Health Sciences, University of Cape Town, South Africa
}

Corresponding author: K G H Katundu (kkatundu@gmail.com)

\begin{abstract}
Background. Septic shock is associated with endothelial dysfunction and oxidative stress, against which vitamin C plays a protective role, possibly influencing clinical outcome. Hyperglycaemia may lower vitamin C.

Objective. To study plasma vitamin C, oxidative stress, hyperglycaemia, endothelial dysfunction and outcome in septic shock.

Methods. In a prospective, observational study of 25 adult septic shock patients, serial blood samples were analysed for vitamin C, thiobarbituric acid-reactive substances (TBARS) (a biomarker of oxidative stress), and soluble vascular cell adhesion molecule-1 (sVCAM-1) and E-selectin (markers of endothelial dysfunction). Blood glucose, Sequential Organ Failure Assessment (SOFA) scores and fluid requirements were monitored. Results. Plasma vitamin C was low, while plasma TBARS were high throughout the 7-day study period. Endothelial dysfunction markers (sVCAM-1 and E-selectin) were high at the baseline. VCAM-1 decreased significantly on day 1 and normalised on day 7. E-selectin was unchanged on day 1 compared with baseline, but increased significantly on day 7. Oxidative stress and endothelial dysfunction were associated with increased SOFA score. Increased oxidative stress was associated with increased requirements for intravenous fluids and prolonged duration of vasoconstrictor support. Nine patients died in hospital. At baseline, levels of TBARS were significantly higher in non-survivors than in the survivors of septic shock. Conclusion. In septic shock, clinically relevant oxidative stress was associated with endothelial dysfunction, low vitamin $\mathrm{C}$ and high glucoseto-vitamin-C ratios. Markers of oxidative stress and endothelial damage were increased and correlated with resuscitation fluid requirements, vasoconstrictor use, organ failure and mortality.
\end{abstract}

S Afr J Crit Care 2016;32(1):21-27. DOI:10.7196/SAJCC.2016.v32i1.270

The third international consensus definition for sepsis and septic shock describes septicshockas'a subset of sepsisin which underlying circulatory, cellular, and metabolic abnormalities are associated with a greater risk of mortality than sepsis alone. ${ }^{[1]}$ Microcirculatory dysfunction is the hallmark of septic shock and its persistence is associated with poor outcome. ${ }^{[2]}$ Endothelial function and the glycocalyx are deranged, resulting in increased permeability and poor reactivity to vasoactive substances. ${ }^{[3-5]}$ Septic shock is accompanied by intense oxidative stress arising from the increased production of free radicals under conditions of inadequate antioxidative defences. ${ }^{[6]}$ Uncontrolled oxidative stress has been associated with organ dysfunction and multiorgan failure.$^{[7]}$ It has been suggested that management targeting the microcirculation, distinct from the macrocirculation, could potentially decrease the burden of organ failure in sepsis. ${ }^{[8]}$

Antioxidant defences during severe sepsis may be important. Vitamin $\mathrm{C}$, an antioxidant that works as the first line of defence against free radical action in plasma, is considered essential for normal endothelial function. ${ }^{[0]}$ Vitamin C requirement may be increased in septic shock following losses due to increased oxidative stress, redistribution of blood volume from the intravascular to the extravascular space, and urinary losses or dialysis. . $^{[4,7,10]}$

In the endothelial cells, depletion of vitamin C may be induced in part by the intense hyperglycaemia typical of septic shock. Not only does hyperglycaemia enhance urinary losses of vitamin $C^{[11]}$ but, owing to the structural similarity between glucose and dehydroascorbic acid - one of the forms of vitamin C - glucose competitively inhibits the uptake of dehydroascorbic acid by the endothelial common glucose transporters, GLUT1 and GLUT3 ${ }^{[9]}$ Therefore, the ratio of plasma glucose to vitamin C may be important.

In this study we investigated the possibility of a link between plasma vitamin $\mathrm{C}$ levels and oxidative stress, endothelial dysfunction and clinical markers in patients with septic shock.

\section{Methods}

This prospective study was conducted in the intensive care unit (ICU) of a tertiary academic hospital. Patients with septic shock expected to survive more than 24 hours were enrolled within 12 hours of the commencement of vasoconstrictor support. Septic shock was defined as two or more systemic inflammatory response syndrome criteria, a proven or presumed source of sepsis and a systolic blood pressure of $<90 \mathrm{mmHg}$, or the need for vasoconstrictors after adequate volume resuscitation. Exclusion criteria applied at screening included the following: underweight (body mass index $(\mathrm{BMI})<18 \mathrm{~kg} / \mathrm{m}^{2}$ ), patients unlikely to survive more than 24 hours, parenteral nutritional support, pregnancy, gastrointestinal fistula or other considerable exudative losses, renal dialysis, or more than $100 \mathrm{mg}$ vitamin C supplementation during the previous 7 days. The study was approved by the Human Research Ethics Committee of the Faculty of Health Sciences, University of Cape Town (UCT) (Ref. UCT/FHS/HREC 528/2011). Due 
to the observational nature of the study and the minimal risk to the participants, we were permitted to utilise a deferred informed consent procedure where patients were enrolled in the study when they met the eligibility criteria. Blood samples and collected data were stored until the patient was well enough to give written informed consent for the use of such data. If the patient declined participation, all data and derived data were excluded from the study and destroyed. If a patient died before deferred written informed consent could be obtained, informed consent was waived and we were permitted to use the data.

The patients were managed according to the Surviving Sepsis Campaign international guidelines. ${ }^{[12]}$ Crystalloid fluids were used for resuscitation using dynamic endpoints, and adrenaline was used as the vasoconstrictor. An insulin infusion was used if blood glucose exceeded $10 \mathrm{mmol} / \mathrm{L}$. No vitamin C was administered during the study period. Clinical, demographic and outcome data were collected from the patients' clinical records and from their charts during their admission. Sequential Organ Failure Assessment (SOFA) was scored daily, Acute Physiology and Chronic Health Evaluation (APACHE II) levels were calculated from data collected in the first 24 hours of admission, and daily mean blood glucose and ranges were taken from the clinical records. Blood sampling for plasma thiobarbituric acid-reactive substances (TBARS), vitamin C, soluble vascular cell adhesion molecule-1 (sVCAM-1) and E-selectin was done on enrolment and thereafter daily until vasoconstrictor cessation and on the 7th day following cessation of vasoconstrictors. For each assay, $5 \mathrm{~mL}$ of whole blood was drawn into a chilled heparinised/ ethylenediaminetetraacetic acid (EDTA) vacutainer, which was kept on ice for transport to the laboratory. All samples were centrifuged at $1000 \mathrm{rpm}$ for 10 minutes, and the plasma was drawn off into $1.5 \mathrm{~mL}$ Eppendorf (Axygen Inc., US) tubes, which were coded and stored at -20 $-80^{\circ} \mathrm{C}$ until batch analysis.

The TBARS assay was performed according to a modified method devised by Jentzsch et al. ${ }^{[13]}$ and then developed and optimised at the Lipidology Research Laboratory, UCT. Plasma concentration of vitamin $\mathrm{C}$ was determined using a ferric reducing ascorbate (FRASC) assay kit (\#K671-100, BioVision Research Products, USA). The sVCAM-1 was determined using a commercial human sVCAM-1 enzyme-linked immunosorbent assay (ELISA) kit (RayBiotech, USA). ELISA for E-selectin was performed using the Human E-selectin ELISA kit (RayBiotech, USA). Blood glucose values were obtained from the routine ICU blood glucose monitoring assessments.

Using an alpha error of $5 \%$ and power of $80 \%$, the sample size required to detect at least a $40 \%$ prevalence of deficient vitamin $\mathrm{C}$ status was calculated to be 23 participants. Statistical analysis of the data was done using Stata version 12 (StataCorp, USA) and Statistica version 11 (Statsoft, USA). The Shapiro-Wilks normality test was used to test the data for normality. Descriptive statistics were expressed as mean (SD) and median (IQR) for the continuous data, depending on whether the data were parametric or non-parametric. The Wilcoxon rank-sum test (or Mann-Whitney $U$-test) was used to test the null hypothesis that two populations (survivors and non-survivors of septic shock or males and females) had equal medians in terms of investigating differences in the measured variables. Testing the differences between the means and the medians of the measured variables at different points in time (i.e. the baseline (day 0 ), day 1 and day 7), the Wilcoxon rank-sum test and the repeated measures for analysis of variance (ANOVA) or the Friedman test (K-related samples) were used. To measure the associations between the variables vitamin C, TBARS, sVCAM-1, E-selectin and the clinical outcomes of interest, Spearman's test of association was used. A $p$-value of $<0.05$ was considered statistically significant.

\section{Results}

Eighty patients were screened and 25 sequential patients who fitted the study criteria were enrolled (Fig. 1).

The baseline characteristics of the study population are given in Table 1. The mean (SD) baseline SOFA score was significantly lower in survivors compared with non-survivors of septic shock (9.8 (2.7) v. $12.2(2.1), p=0.014)$.

The median (IQR) vitamin $C$ levels were low compared with the normal reference range $(11-114 \mathrm{nmol} / \mathrm{mL})^{[14]}$ with no significant

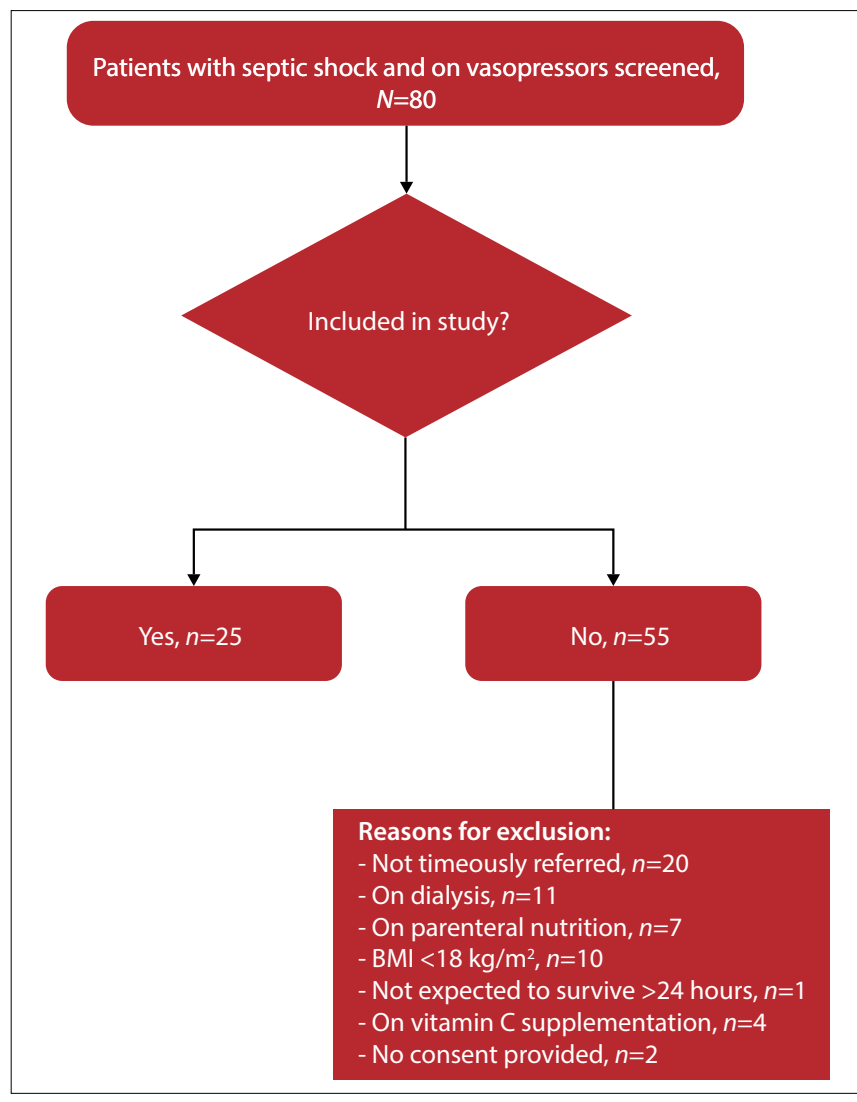

Fig. 1. Flowchart of patients who met inclusion/exclusion criteria for the study population.

Table 1. Characteristics of the study population $(N=25)$

\begin{tabular}{ll}
\hline Gender, $n$ (\%) & $7(28)$ \\
Male & $18(72)$ \\
Female & $49.0(15.6)$ \\
Age (years), mean (SD) & \\
Source of sepsis, $n$ (\%) & $8(32)$ \\
Intra-abdominal & $7(28)$ \\
Respiratory & $7(28)$ \\
Necrotising fasciitis and soft tissue & $3(12)$ \\
Polytrauma with secondary infection & $28.2(6.4)$ \\
BMI (kg/m²), mean (SD) & $20.1(10.5)$ \\
APACHE II score, mean (SD) & $10.6(2.8)$ \\
Baseline SOFA score, mean (SD) & $54.9(10.9)$ \\
Lowest MAP (mmHg), mean (SD) & $16(64)$ \\
Survivors, $n$ (\%) & $6(4-10)$ \\
Length of ICU stay (days), median (IQR) & \\
MAP = mean arterial pressure. &
\end{tabular}


change from baseline to day 7 (baseline: 5.7 (2.3 - 8.0) nmol/mL, day 1: $5.9(3.7-14.2)$ $\mathrm{nmol} / \mathrm{mL}$, day 7: $5.6(3.7-9.4) \mathrm{nmol} / \mathrm{mL}$, $p=0.83$ ). Compared with the normal reference range of $1.9-3.9 \mathrm{nmol} / \mathrm{mL}^{[15]}$ the median TBARS level in the patients as a marker of oxidative stress was high at baseline (19.5 (14.0 - 37.0) nmol/mL) and continued to be high at day $1(20.4(13.0-64.0) \mathrm{nmol} / \mathrm{mL})$, with no statistically significant reduction at day $7(13.3(9.0-18.0) \mathrm{nmol} / \mathrm{mL}, p=0.52)$. At baseline, non-survivors of septic shock had higher median TBARS levels (mmol/ $\mathrm{mL})$ than survivors $(16.9(11.9-21.7)$ v. 43.8 (23.6 - 47.7) $\mathrm{mmol} / \mathrm{mL}, p=0.008$ ).

The median (IQR) plasma sVCAM-1 levels were raised at baseline and day 1 but decreased significantly at day $7(p<0.001)$ (Fig. $2 \mathrm{~A})$. When further analysed, the SVCAM- 1 levels at baseline in survivors were significantly lower than those of non-survivors (Fig. 2B).

Fig. 3A indicates the elevated median (interquartile range (IQR)) plasma E-selectin levels at baseline. The E-selectin levels significantly increased from day 1 to day 7 $(p=0.003)$. Comparing survivors and nonsurvivors at baseline, the levels were found to be significantly higher in survivors than nonsurvivors (13.1 (7.8 - 24.1) v. $7.1(6.2-15.3)$ $\mathrm{ng} / \mathrm{mL}, p=0.04)$ (Fig. 3B).

Since oxidative stress may influence endothelial dysfunction, the association between TBARS as a marker of oxidative stress and the biomarkers of endothelial function was tested. These results are presented in Table 2. A moderate but significant positive correlation was found between SVCAM- 1 and TBARS at all the time points. However, no significant correlation was observed between E-selectin and TBARS at any of the time points.

The median (IQR) blood glucose from the routine records was highest at baseline ( 8.8 (7.3 - 10.1) $\mathrm{mmol} / \mathrm{L}$ ), but decreased to within the ICU target range of $5-8 \mathrm{mmol} / \mathrm{L}$ on the subsequent research days (day 1: 7.8 (6.0 - 8.6) $\mathrm{mmol} / \mathrm{L}$, day 7: $7.2(6.4-7.8 \mathrm{mmol} / \mathrm{L})$. Similarly, intravenous fluid volume infused per 24 hours in the patients was highest at baseline (1 870 (1 410 - 2 615) $\mathrm{mL}$ ) but decreased significantly on day $1(1046(500-1680) \mathrm{mL}, p=0.0004)$ and did not change significantly at day 7 (704 (0 - 2 250) $\mathrm{mL}, p=0.7$ ). The median (IQR) duration on vasoconstrictors during ICU admission was 30 (24 - 38) hours.

Fig. 4 indicates the association between oxidative stress and intravenous fluid requirements at baseline and inotrope-free days at day 7. It was found that increased

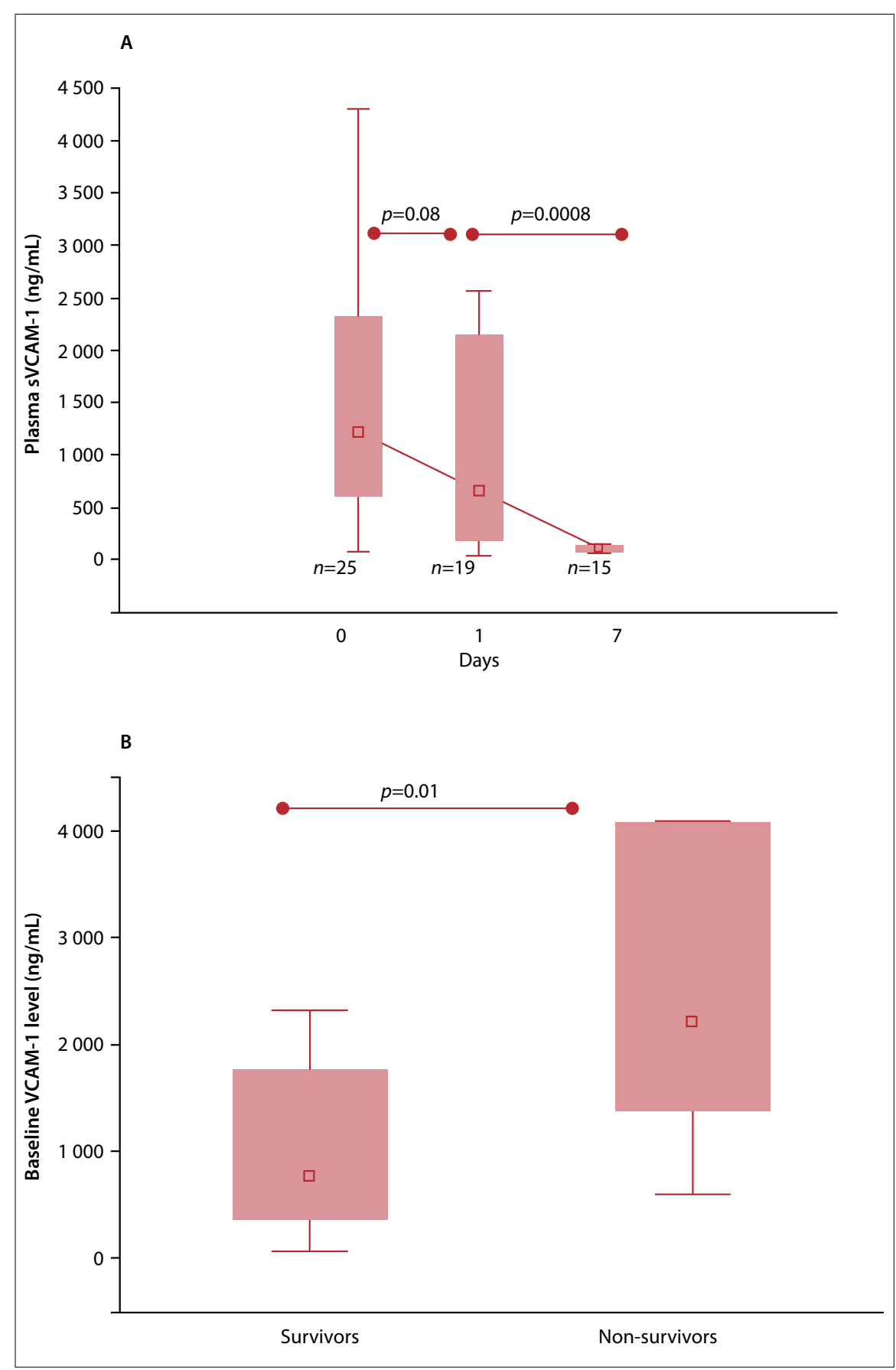

Fig. 2. sVCAM-1 levels in the patients. (A) Plasma sVCAM-1 concentrations over the study period. At baseline, the SVCAM-1 levels were higher than reference values with no statistically significant change at day 1 but significantly decreased from day 1 to day 7. (B) Comparison of baseline sVCAM1 between survivors and non-survivors. Non-survivors of septic shock had statistically significantly higher levels at baseline than survivors. Normal reference range is $72-349 \mathrm{ng} / \mathrm{mL}$.

oxidative stress was associated with increased requirement for intravenous fluid at baseline and fewer inotrope-free days.

The associations between oxidative stress (TBARS) and severity of illness as indicated by SOFA score, and between SOFA and SVCAM-1 scores, were also determined (Fig. 5). A positive correlation was found between TBARS and SOFA score. Baseline high sVCAM-1 levels were also associated with high SOFA scores.
The ratio of glucose to vitamin $\mathrm{C}$ was calculated and found to be high at all time points compared with the expected reference physiological ratio, with no statistically significant change over the period (Fig. 6).

\section{Discussion}

This is the first study in a South African (SA) setting to investigate vitamin C status, oxidative stress levels, hyperglycaemia and 


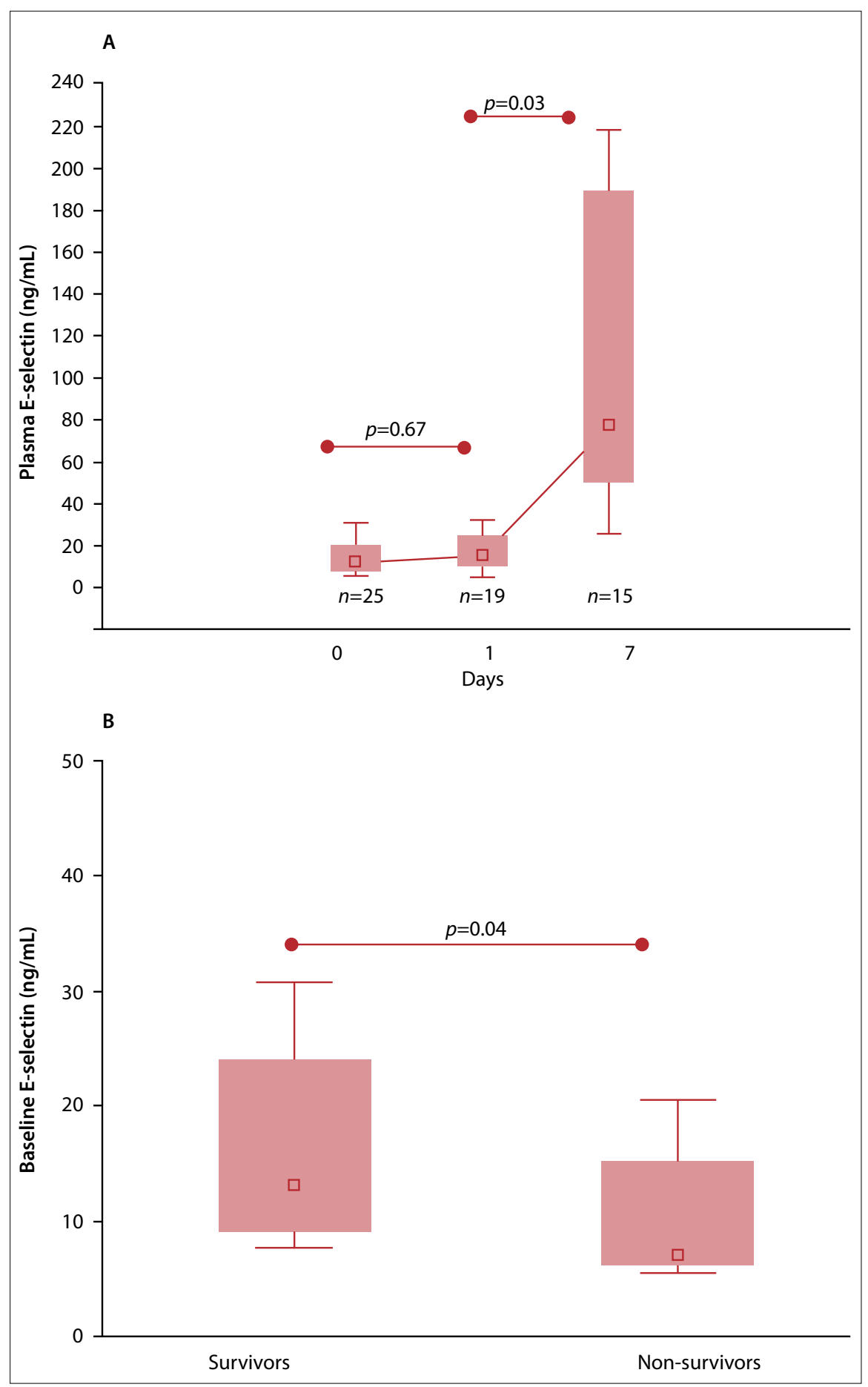

Fig. 3. E-selectin levels in the patients. (A) shows plasma E-selectin concentrations over the study days. E-selectin levels were higher than reference range at baseline and day 1 but increased significantly at day 7. (B) shows the comparison of baseline plasma E-selectin levels between survivors and non-survivors at baseline. At baseline, survivors had statistically significantly higher values than non-survivors. Normal reference range for E-selectin is $0.9-2.8 \mathrm{ng} / \mathrm{mL}$.

Table 2. Association between endothelial function markers and TBARS as a marker of oxidative stress

\begin{tabular}{|c|c|c|c|c|}
\hline \multirow[b]{2}{*}{ Study day } & \multicolumn{2}{|c|}{ sVCAM-1 v. TBARS } & \multicolumn{2}{|c|}{ E-selectin v. TBARS } \\
\hline & Spearman's rho & $p$-value & Spearman's rho & $p$-value \\
\hline Baseline & 0.45 & 0.02 & 0.08 & 0.70 \\
\hline Day 1 & 0.57 & 0.01 & 0.16 & 0.50 \\
\hline
\end{tabular}

their association with endothelial dysfunction in patients with septic shock. This study has shown that septic shock is associated with low levels of plasma vitamin $C$, which persisted until day 7 after the cessation of inotropic support. There was also evidence of oxidative stress in the patients, marked by increased levels of TBARS, and there was no significant reduction in these levels at day 7 of the study. Non-survivors had increased levels of oxidative stress and organ failure compared with survivors. The results also showed that the plasma glucoseto-vitamin-C ratios were higher than the normal expected ratio on all of the study days. Both plasma biomarkers of endothelial dysfunction (sVCAM-1 and E-selectin) were high at baseline. However, sVCAM-1 levels were significantly higher in non-survivors than in survivors, although this difference was not observed for E-selectin levels. Furthermore, the sVCAM-1 levels fell significantly at day 1 , and normalised at day 7, while the E-selectin levels were constantly raised at day 1 , as at baseline, and further increased significantly at day 7. Both sVCAM- 1 and TBARS associated positively to SOFA score, and increased TBARS levels were associated with increased requirements for intravenous fluids for resuscitation and an increased number of days on vasoconstrictors.

The study group had extremely low vitamin C levels, with plasma levels reaching only approximately $50 \%$ of the lower limit of reference range. These levels are comparable with those reported in a similar study where, without intervention, plasma ascorbic acid levels were borderline normal or below normal reference values at baseline and continued to decline during ICU admission, in a similar patient population. ${ }^{[16,17]}$ There are a number of reasons for such low levels. Firstly, there is profound oxidative stress and consequent antioxidant utilisation associated with critical illness, ${ }^{[6,7,16]}$ and excess losses of plasma antioxidants via circulating volume redistribution to the extravascular space. Secondly, although study patients received enteral nutritional support, it has previously been shown that it is not possible to restore normal plasma levels while in ICU through nutritional support alone, without the addition of parenteral high-dose vitamin C. ${ }^{[18]}$ Thirdly, in our patient collective, the very low levels could be due to low plasma vitamin $\mathrm{C}$ prior to the onset of septic shock, since low vitamin C status is known to be common in the healthy SA population owing to low micronutrient intake. ${ }^{[19,20]}$ This patient population may, therefore, be predisposed to low vitamin $\mathrm{C}$ levels in acute illness because of a pre-existing 
nutritional compromise in the diet, which is worsened by the increased demands during septic shock. ${ }^{[21]}$

Patients also had high plasma glucose-tovitamin-C ratios, above the reference value of 88 calculated from the expected physiological levels of both glucose and vitamin C. The increased ratio was mainly contributed to by the very low plasma vitamin $\mathrm{C}$ levels and to a lesser extent the high blood glucose levels at baseline. This unfavourably high ratio could partly explain the increased oxidative stress through the glucose-mediated induction of inflammatory response, ${ }^{[22,23]}$ as well as competitive inhibition of cellular vitamin C uptake. ${ }^{[22,24]}$ Lax management of sepsisrelated stress hyperglycaemia together with the common clinical practice of administering systemic steroids in vasopressor-dependent patients, which worsens glucose control, may contribute to oxidative stress in this population.

Importantly, TBARS levels at baseline were positively associated with increased organ dysfunction and/or failure, as measured by SOFA score, and an increase in sVCAM-1 (a marker of endothelial damage). The association between oxidative stress and increased endothelial and organ dysfunction in this study is of clinical relevance. Previous literature has shown that oxidative stress causes damage to the endothelial glycocalyx. ${ }^{[25]}$ Damage to the glycocalyx disrupts its shielding function to the vascular walls from direct exposure to blood flow, while serving as a vascular permeability barrier. ${ }^{[26]}$ Disruption of the glycocalyx therefore causes capillary leakage and predisposes the endothelial cells to oxidative stress-induced disruption, when endothelial cell tight junctions may be damaged by mechanisms such as protein modification, thiol-oxidation, phosphorylation, nitration and carboxylation during sepsis. ${ }^{[27]}$ Endothelial dysfunction in sepsis has been demonstrated to cause microvascular dysfunction, the motor for sepsis-induced organ dysfunction and failure that may be reflected by SOFA score. ${ }^{[28-30]}$ The baseline oxidative stress marker in this study was significantly higher in the non-survivors, who also had increased organ dysfunction when compared with survivors. These results, therefore, demonstrate that increased oxidative stress is a marker of increased risk of death; this finding supports previous similar findings of other researchers. ${ }^{[31,32]}$

Contrary to the hypothesis, no significant correlation was found between E-selectin and TBARS. The lack of correlation could be a consequence of the effect of increased oxidative damage to the endothelial cells, a factor known

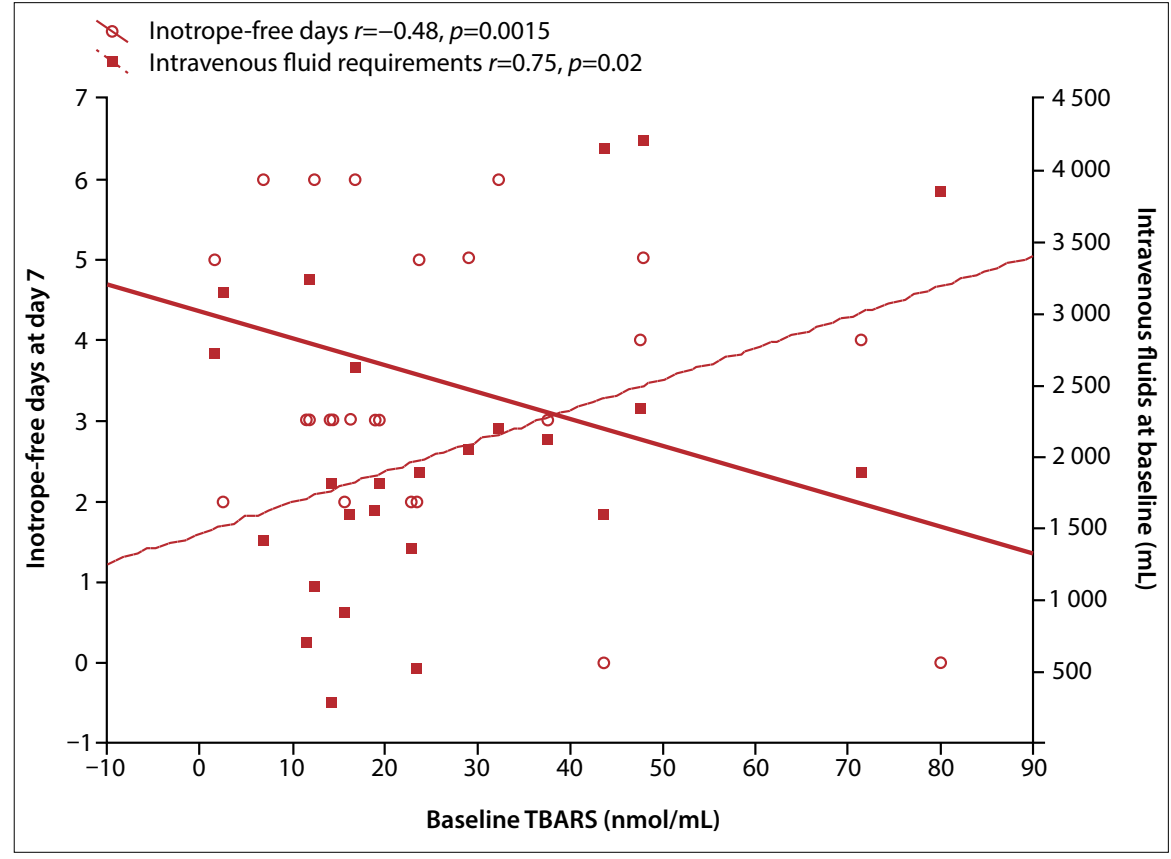

Fig. 4. Correlation between baseline TBARS and inotrope-free days at day 7, and baseline TBARS and baseline intravenous fluid requirements.

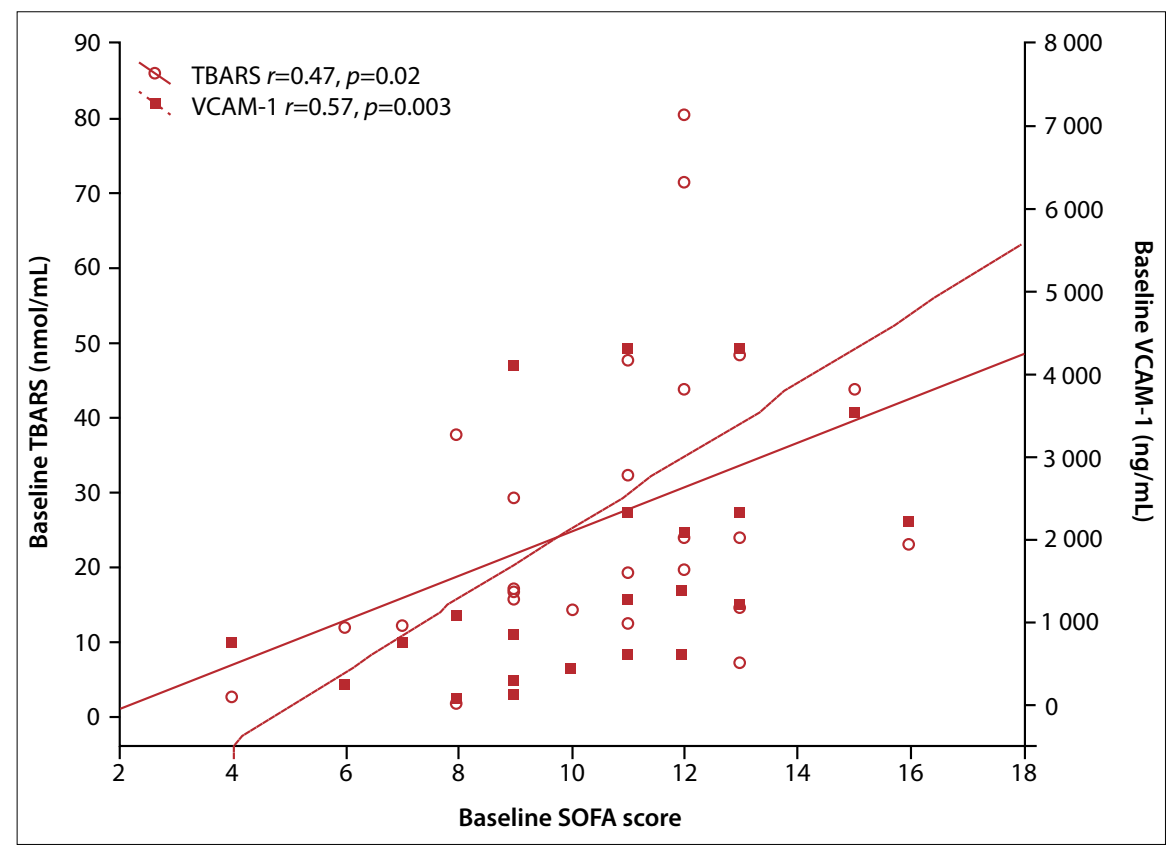

Fig. 5. Correlation between baseline TBARS and SOFA score, baseline SVCAM-1 and SOFA score.

to negatively affect E-selectin expression. ${ }^{[33]}$ This would also explain the findings of this study that E-selectin levels were higher in the survivors than in the non-survivors. The non-survivors of septic shock in the current study had increased levels of oxidative stress, with increased levels of sVCAM-1, marking endothelial damage. Since endothelial cell damage and necrosis do not induce E-selectin expression, ${ }^{[34,35]}$ it is not unexpected to find higher E-selectin levels in the survivors, who may have relatively less endothelial damage than the non-survivors. Further to this, it has been suggested that increased E-selectin levels may reduce collateral damage to the host. ${ }^{[35]}$ Indeed, one study in children with sepsis found that those with the highest levels of E-selectin exhibited the best outcome and survival rates. ${ }^{[36]}$

In survivors, sVCAM-1 levels decreased at day 1 and decreased to within a normal range within 7 days of stopping vasoconstrictors. This demonstrates that there was less cellular damage and recovery in endothelial functions, as septic shock resolved. This was an expected result. However, the plasma E-selectin levels remained relatively stable from baseline to 


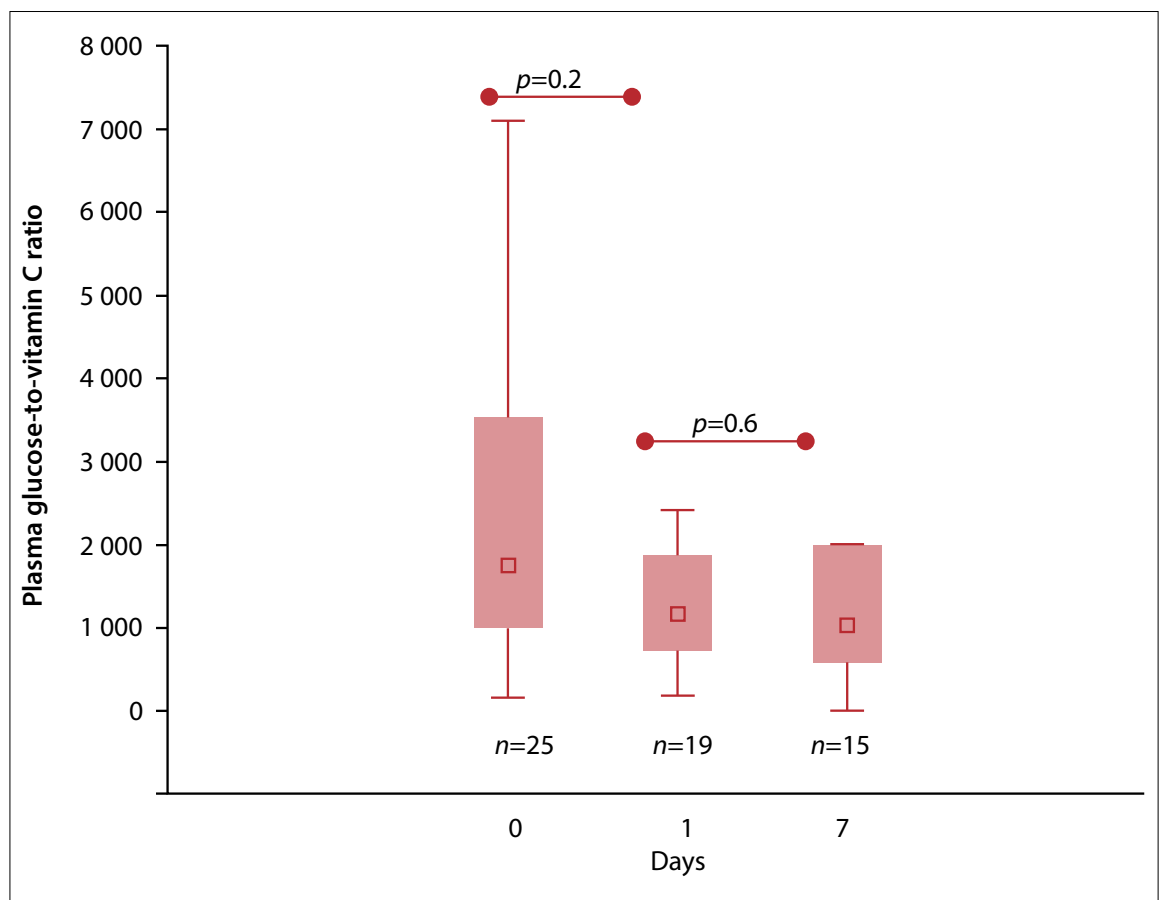

Fig. 6. Glucose-to-vitamin-C ratios over the study period.

day 1 but increased significantly at day 7 . It is known that in addition to being stimulated by inflammatory mediators, increased E-selectin expression has been described as a marker of proliferating endothelium and is known to be involved in angiogenesis. ${ }^{[37-39]}$ Increased E-selectin expression at day 7 may therefore reflect increased endothelial proliferation in repair and regeneration, post resolution of septic shock.

This study has demonstrated that increased oxidative stress at baseline is associated with an increased requirement for intravenous fluids for resuscitation and more days on inotropic support. One of the major organs affected in septic shock is the cardiovascular system and it is not surprising that such patients require large amounts of intravenous fluids for resuscitation and inotropic support, to restore macrovascular haemodynamics. ${ }^{[12]}$ It may be hypothesised that reducing oxidative stress may result in the reduction of intravenous fluid requirements in this patient group. A randomised study of vitamin C supplementation in burns patients ${ }^{[40]}$ demonstrated that high doses of vitamin $\mathrm{C}$ resulted in reduced oxidative stress levels and lower requirements of resuscitation fluids in the patients who received vitamin C supplementation, compared with controls. Although septic shock and burns are different disease conditions, they have common factors, as they both exhibit a profound inflammatory response and are associated with increased oxidative stress. A recent review on the possible use of vitamin $\mathrm{C}$ in critically ill research to focus on the prompt use of shortterm, high-dose intravenous vitamin $\mathrm{C}$ as a resuscitation drug. ${ }^{[41]}$ Vitamin $\mathrm{C}$ may intervene in the oxidant cascade for optimisation of the macro- and microcirculation and limitation of cellular injury. ${ }^{[41]}$

Another interesting finding of this study is that increased oxidative stress was associated with more days on inotropic support. This demonstrates that increased oxidative stress in septic shock is somehow associated with circulatory dysfunction, so that this patient group requires more time on vasoconstrictors, presumably due to lingering endothelial dysfunction. Use of vasoconstrictors is very important clinically because it reflects illness severity and, therefore, the need for organ support and ICU resources. Early liberation from vasoconstrictors is an indication of an accelerated improvement and response to the other critical aspects of sepsis control such as source control and antibiotic responsiveness.

\section{Study limitations}

Limitations of this study include its design as a cross-sectional study. The study can only report associations between variables and cannot demonstrate any cause-and-effect relationship. Our sample size was 25 patients; although a relatively small sample, we met the sample size required for the planned statistical analysis. Because of the study design, in vivo assessment procedures were not indicated for assessment of the endothelium and the use of circulating biomarkers was considered a suitable proxy for the observational purposes of this baseline study. This study was conducted prior to the publication of the new consensus definition of septic shock, but the majority of our participants also complied with this definition.

\section{Conclusions}

The results of the study demonstrate clinically relevant oxidative stress-associated endothelial dysfunction in a context of profoundly low vitamin $\mathrm{C}$ plasma levels and high plasma glucose-to-vitamin-C ratios. Markers of oxidative stress and endothelial damage were increased and correlated with resuscitation fluid requirements, vasoconstrictor use, organ failure and death. Strategies to limit oxidative damage in septic shock, such as short-term, high-dose vitamin C supplementation, merit further research attention.

Acknowledgements. We acknowledge Shaheen Mowla, Dee Blackhurst and Fahima Adams for their assistance with the laboratory aspects of the study, and the SA Consortium for Research Excellence (SACORE) and UCT for financial support.
References

1. Singer M, Deutschman CS, Seymour CW, et al. The Third International Consensus Definitions for Sepsis and Septic Shock (Sepsis-3). JAMA 2016;315(8):801-810. DOI:10.1001/ jama.2016.0287

2. Hernandez G, Bruhn A, Ince C. Microcirculation in sepsis New perspectives. Curr Vasc Pharmacol 2013;11(2):161-169. DOI:10.2174/1570161111311020006

3. De Backer D, Orbegozo Cortes D, Donadello K, Vincent J. Pathophysiology of microcirculatory dysfunction and the pathogenesis of septic shock. Virulence 2014;5(1):73-79. DOI:10.4161/viru.26482

4. Wilson JX. Evaluation of vitamin C for adjuvant sepsis therapy. Antioxid Redox Signal 2013;19(17):2129-2140. DOI:10.1089/ars.2013.5401

5. Donati A, Damiani E, Botticelli L, et al. The aPC treatment improves microcirculation in severe sepsis/ DOI:10.1186/1471-2253-13-25

6. Doise J, Aho LS, Quenot J, et al. Plasma antioxidant status in septic critically ill patients: A decrease over time. Fundam Clin Pharmacol 2008;22(2):203-209. DOI:10.1111/j.14728206.2008.00573.x

7. Heyland D, Muscedere J, Wischmeyer PE, et al. A randomized trial of glutamine and antioxidants in critically ill patients. N Engl J Med 2013;368(16):1489-1497. DOI:10.1056 NEJMoa1212722

8. Trzeciak S, McCoy JV, Phillip Dellinger R, et al. Early increases in microcirculatory perfusion during protocoldirected resuscitation are associated with reduced multiorgan failure at $24 \mathrm{~h}$ in patients with sepsis. Intensive Care Med 2008;34(12):2210-2217. DOI:10.1007/s00134-0081193-6

9. Wilson JX. Mechanism of action of vitamin C in sepsis: Ascorbate modulates redox signaling in endothelium. Biofactors 2009;35(1):5-13. DOI:10.1002/biof.7

10. Raimann JG, Levin NW, Craig RG, Sirover W, Kotanko P, Handelman G. Is vitamin C intake too low in dialysis patients? Semin Dial 2013;26(1):1-5. DOI:10.1111/sdi.12030

11. Will JC, Byers T. Does diabetes mellitus increase the requirement for vitamin C? Nutr Rev 1996;54(7):193-202. DOI:10.1111/j.1753-4887.1996.tb03932.x

12. Dellinger RP, Levy MM, Rhodes A, et al. Surviving sepsis campaign: international guidelines for management of severe sepsis and septic shock: 2012. Crit Care Med 2013;41(2):580637. DOI:10.1097/CCM.0b013e31827e83af

13. Jentzsch AM, Bachmann H, Fürst P, Biesalski HK. Improved analysis of malondialdehyde in human body fluids. Free Radic Biol Med 1996;20(2):251-256. DOI:10.1016/08915849(95)02043-8 
14. Blanchard J. Depletion and repletion kinetics of vitamin C in humans. J Nutr 1991;121(2):170-

15. Miller E, Markiewicz L, Saluk J, Majsterek I. Effect of short-term cryostimulation on antioxidative status and its clinical applications in humans. Eur J App Physiol 2012;112(5):1645-1652. DOI:10.1007/s00421-011-2122-x

16. Long CL, Maull KI, Krishnan RS, et al. Ascorbic acid dynamics in the seriously ill and injured. J Surg Res 2003;109(2):144-148. DOI:10.1016/S0022-4804(02)00083-5

17. Nathens $A B$, Neff MJ, Jurkovich GJ, et al. Randomized, prospective trial of antioxidan supplementation in critically ill surgical patients. Ann Surg 2002;236(6):814-822 DOI:10.1097/00000658-200212000-00014

18. Berger MM. Vitamin C requirements in parenteral nutrition. Gastroenterology 2009;137(5 Suppl):S70-S78. DOI:10.1053/j.gastro.2009.08.012

19. Segal I, Gut A, Schofield D, Shiel N, Braganza JM. Micronutrient antioxidant status in black South Africans with chronic pancreatitis: Opportunity for prophylaxis. Clin Chim Acta 1995;239(1):7179. DOI:10.1016/0009-8981(95)06102-J

20. MacIntyre UE, Kruger HS, Venter CS, Vorster HH. Dietary intakes of an African population in different stages of transition in the North West Province, South Africa: The THUSA study. Nutr Res 2002;22(3):239. DOI:10.1016/S0271-5317(01)00392-X

21. Berger MM. Antioxidant micronutrients in major trauma and burns: Evidence and practice. Nut Clin Pract 2006;21(5):438-449. DOI:10.1177/0115426506021005438

22. Ceriello A, Esposito K, Ihnat M, Thorpe J, Giugliano D. Effect of acute hyperglycaemia, long-term glycaemic control and insulin on endothelial dysfunction and inflammation in Type 1 diabetic patients with different characteristics. Diabet Med 2010;27(8):911-917. DOI:10.1111/j.14645491.2009.02928.x

23. Gordin D, Forsblom C, Rönnback M, et al. Acute hyperglycaemia induces an inflammatory response in young patients with type 1 diabetes. Ann Med 2008;40(8):627-633. DOI:10.1080/07853890802126547

24. Dungan KM, Braithwaite SS, Preiser J. Stress hyperglycaemia. Lancet 2009;373(9677):1798-1807. DOI:10.1016/S0140-6736(09)60553-5

25. Marechal X, Favory R, Joulin O, et al. Endothelial glycocalyx damage during endotoxemia coincides with microcirculatory dysfunction and vascular oxidative stress. Shock 2008;29(5):572 576. DOI:10.1097/shk.0b013e318157e926

26. Van den Berghe G, Wilmer A, Hermans G, et al. Intensive insulin therapy in the medical ICU. N Engl J Med 2006;354(5):449-461. DOI:10.1056/NEJMoa052521

27. Rao R. Oxidative stress-induced disruption of epithelial and endothelial tight junctions. Fron Biosci 2008;13:7210-7226. DOI:10.2741/3223
28. Gomez H, Ince C, De Backer D, et al. A unified theory of sepsis-induced acute kidney injury: Inflammation, microcirculatory dysfunction, bioenergetics, and the tubular cell adaptation to injury. Shock 2014;41(1):3-11. DOI:10.1097/SHK.0000000000000052

29. Piagnerelli M, Ince C, Dubin A. Microcirculation. Crit Care Res Pract 2012;867176. DOI:10.1155/2012/867176

30. Ren J. Wide spectrum of presentation and variable mechanisms of compromised cardiac unction in multiple organ dysfunction syndrome. J Organ Dysfunct 2008;4(4):239-248. DOI:10.1080/17471060701200394

31. Costa NA, Gut AL, De Souza D, et al. Serum thiamine concentration and oxidative stres as predictors of mortality in patients with septic shock. J Crit Care 2013;29(2):249-252 DOI:10.1016/j.jcrc.2013.12.004

32. Huet $\mathrm{O}$, Obata R, Aubron C, et al. Plasma-induced endothelial oxidative stress is related to the severity of septic shock. Crit Care Med 2007;35(3):821-826. DOI:10.1097/01.ccm.0000257464.79067.af

33. Harrington EO, Stefanec T, Newton J, Rounds S. Release of soluble E-selectin from activated endothelial cells upon apoptosis. Lung 2006;184(5):259-266. DOI:10.1007/s00408-005-2589-5

34. Pigott R, Dillon LP, Hemingway IH, Gearing AJ. Soluble forms of E-selectin, ICAM-1 and VCAM-1 are present in the supernatants of cytokine activated cultured endothelial cells. Biochem Biophys Res Commun 1992;187(2):584-589. DOI:10.1016/0006-291x(92)91234-h

35. Zonneveld R, Martinelli R, Shapiro NI, Kuijpers TW, Plötz FB, Carman CV. Soluble adhesion molecules as markers for sepsis and the potential pathophysiological discrepancy in neonates children and adults. Crit Care 2014;18(1):204. DOI:10.1186/cc13733

36. Briassoulis G, Papassotiriou I, Mavrikiou M, Lazaropoulou C, Margeli A. Longitudinal course and clinical significance of TGF-betal, sL- and sE-Selectins and sICAM-1 levels during severe acute tress in children. Clin Biochem 2007:40(5-6):299-304. DOI:10.1016/j.clinbiochem 2006.11.015

7. Kräling BM, Razon MJ, Boon LM, et al. E-selectin is present in proliferating endothelial cells in human hemangiomas. Am J Pathol 1996;148(4):1181-1191.

38. Läubli H, Borsig L. Selectins promote tumor metastasis. Semin Cancer Biol 2010;20(3):169-177. DOI:10.1016/j.semcancer.2010.04.005

39. Smadja DM, Mulliken JB, Bischoff J. E-selectin mediates stem cell adhesion and formation of blood vessels in a murine model of infantile hemangioma. Am J Pathol 2012;181(6):2239-2247. DOI:10.1016/j.ajpath.2012.08.030

40. Tanaka H, Matsuda T, Miyagantani Y, Yukioka T, Matsuda H, Shimazaki S. Reduction of resuscitation fluid volumes in severely burned patients using ascorbic acid administration: $A$ randomized, prospective study. Arch Surg 2000;135(3):326-331. DOI:10.1001/archsurg.135.3.326

41. Oudemans-van Straaten M, Spoelstra-de Man A, De Waard MC. Vitamin C revisited. Crit Care 2014;18(4):460. DOI:10.1186/s13054-014-0460-x 